\title{
HETEROCYCLIC SYNTHESIS USING NITRILIMINES PART 15: SYNTHESIS OF NEW 1,2,4,5-TETRAZINONE AND 1,2,4,5-TETRAZEPINONE DERIVATIVES
}

\author{
Hany M. M. Dalloul
}

\begin{abstract}
Department of Chemistry, Faculty of Applied Science, Al-Aqsa University of Gaza, P.O.Box 4051, Gaza 76888, Palestine. E-mail: hmdalloul60@yahoo.com
\end{abstract}

\begin{abstract}
The reaction of C-aroyl-N-arylnitrilimines II with Girard-reagent $P$ III led to the formation of the 1,2,3,4-tetrahydro-1,2,4,5-tetrazin-3-ones IVa-f and 1,2,6,7tetrahydro-1,2,4,5-tetrazepin-6-ones Va-f, which underwent thermal oxidation to 1,6dihydro-1,2,4,5-tetrazin-6-ones and 6,7-dihydro-1,2,4,5-tetrazepin-6-ones. The microanalysis and spectral data of the synthesized compounds are in full agreement with their molecular structure.
\end{abstract}

\section{KEYWORDS}

Nitrilimines, Cyclization, 1,2,4,5-Tetrazinones, 1,2,4,5-Tetrazepinones.

\section{RESUMO}

A reação de $\mathrm{C}$-aroil- $\mathrm{N}$-arilnitriliminas com o reagente de Girard $P$ III levou a formação dos 1,2,3,4-tetrahidro-1,2,4,5-tetrazino-3-onas IVa-f e 1,2,6,7-tetrahidro-1,2,4,5tetrazepino-6-onas Va-f. Com oxidação térmica formaram 1,6-dihidro-1,2,4,5-tetrazino-6onas e 6,7-dihidro-1,2,4,5-tetrazepino-6-onas. A microanalise e os dados espectrais dos compostos sintetizados concordam plenamente com a estrutura molecular.

\section{PALAVRAS-CHAVE}

Nitriliminas, Ciclização; 1,2,4,5-Tetrazinonas; 1,2,4,5-Tetrazepinonas. 
SOUTH. BRAZ. J. CHEM., Vol.18, No. 18, 2010

\section{INTRODUCTION}

Substituted 1,2,4,5-tetrazines represent significant class of heterocycles that find many useful pharmacological and medicinal applications [1-4]. Azolotetrazinones have been the focus of medicinal chemists in the past decades because of the outstanding antineoplastic activity exhibited by them [5]. Imidazole tetrazinone (Temozolomide: Temodal ${ }^{\circledR}$ ) has shown promising antitumor activity against low and high-grade glioma [3-6], melanoma [7,8], and mycosis fungoides $[8,9]$. Furthermore, some pyridopyrrolotetrazine derivatives showed significant activity against leukemia, non-small lung, colon, central nervous system, melanoma, ovarian, renal, prostate, and breast tumors cell lines. Different methods were used to synthesize 1,2,4,5-tetrazinone derivatives [6], some of which employed the cyclocondensation of nitrilimines, generated in situ from the corresponding hydrazonoyl halides by the action of a suitable base, with nucleophilic substrates incorporating suitably located electrophilic centers. Ottensooser [10] was first reported the synthesis of 1,2,4,6-tetrazepine derivative by the action of dilute potassium hydroxide solution on isobutyl chlorourea. Some derivatives of the fused tetrazepine ring system have been prepared by Sidhu et al. [11] by the treatment of 5-(2-aminophenyl)-4-5,6dihydro-4H-1,2,4,6-tetrazepine with ferric chloride or sodium nitrite solution.

In continuation of our research line dealing with the construction of heterocyclic systems by means of nitrilimines cyclocondensation methodology [7-9], we investigated the reaction of $\mathrm{C}$-substituted $\mathrm{N}$-arylnitrilimines II with acetylhydrazide pyridinium chloride (Girard-reagent P) III in an attempt to synthesize new derivatives of 1,2,4,5-tetrazinones IVa-f and 1,2,4,5-tetrazepin6-ones Va-f in anticipation of expected interesting biological activities.

\section{RESULTS AND DISCUSSION}

Recently we found that 1,1-dimethyl- and 1-methyl-1-phenylhydrazine react readily with nitrilimines, generated in situ from the action of triethylamine onto the hydrazonoyl halides, yielding the corresponding acyclic electrophilic addition products, which cyclized intramolecularly to the corresponding 1,2,3,4tetrahydro-1,2,4,5-tetrazines under thermal conditions [12]. On the other hand, the reaction of the same nitrilimines II with acetylhydrazide pyridinium chloride III (Girard-reagent P) for 12-18 hours in 1,4-dioxane at room temperature gave a cyclocondensation products, 1,2,3,4-tetrahydro-1,2,4,5-tetrazin-3-ones IVa-f and 1,2,6,7-tetrahydro-1,2,4,5-tetrazepin-6-ones Va-f (Figure 1). The purity of the isolated compounds was checked by TLC in different solvents. 


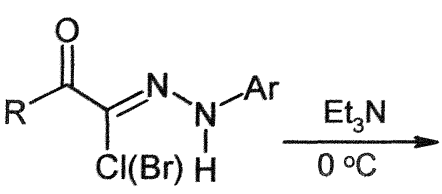

I

$\mathrm{Ar}=4-\mathrm{Cl}-\mathrm{C}_{6} \mathrm{H}_{4}-$

\begin{tabular}{c|cccccc} 
Entry & a & b & c & d & e & f \\
\hline$R$ & $M e$ & $\mathrm{Ph}$ & $\mathrm{PhNH}$ & $2-\mathrm{C}_{4} \mathrm{H}_{3} \mathrm{O}$ & $2-\mathrm{C}_{4} \mathrm{H}_{3} \mathrm{~S}$ & $2-\mathrm{C}_{10} \mathrm{H}_{7}$
\end{tabular}<smiles>[R]C(=O)C1=NN(C)C(=O)NN1</smiles><smiles>NNC(=O)C[n+]1ccccc1Cl</smiles>

III
IVa-f<smiles>[R]C(=O)C1=NN([Al])C(=O)CNN1[Tl]</smiles>

Va-f

Figure 1. Schematic diagram for the synthesis of compounds IVa-f and Va-f

The formation of compounds IVa-f and Va-f is assumed to involve unisolable nucleophilic addition adducts VIII, which undergo intramolecular cyclization in tow pathways as shown in Figure 2. The cyclization at the carbon of amide group (path a) followed with elimination of pyridine methochloride molecule [13] affording the 1,2,3,4-tetrahydro-1,2,4,5-tetrazin-3-one derivatives IVa-f and the cyclization at methylene carbon (path b) followed with loosing pyridine hydrochloride molecule yielding the unknown 1,2,6,7-tetrahydro1,2,4,5-tetrazepin-6-one derivatives Va-f as a minor products.

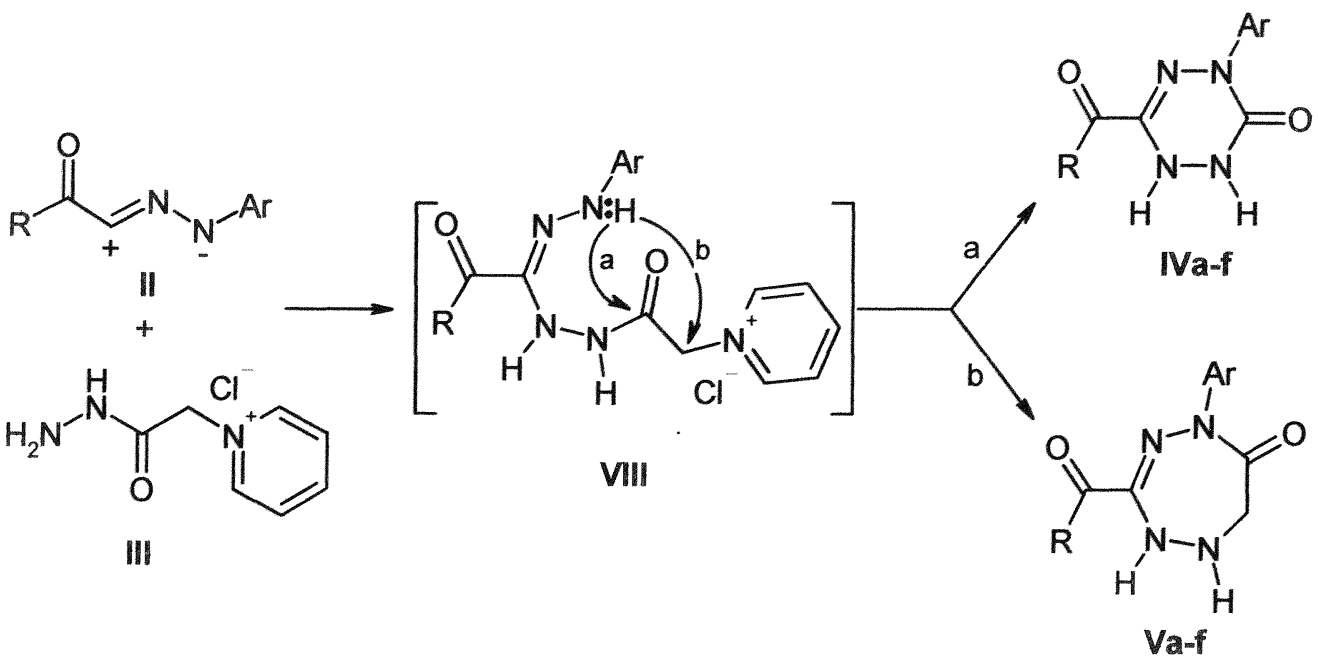

Figure 2. Suggested reaction mechanism 


\subsection{Spectroscopical Data}

The structures of the titled compounds IVa-f and Va-f have been confirmed by elemental analysis and their spectral data (experimental part). Their IR spectra showed intense absorption bands within the $3435-3220 \mathrm{~cm}^{-1}$ range that were attributed to $\mathrm{HN}-\mathrm{NH}$ and $1675-1670 \mathrm{~cm}^{-1}$ assigned to carbonyl of the ring. All these products gave two singlet signals at 6.6-6.4 ppm in their ${ }^{1} \mathrm{H}$ NMR spectra, attributed to the HN-NH protons. In compounds Va-f, the signal of $\mathrm{CH}_{2}$ protons appeared as singlet at $4.3 \mathrm{ppm}$. The ${ }^{13} \mathrm{C}$ NMR spectra of the synthesized compounds IVa-f and Va-f showed all the signals of the proposed structures, specially carbonyl carbon of the ring were found to resonate at about 172 and $171 \mathrm{ppm}$ and the signal at $43.8 \mathrm{ppm}$ are assignable to methylene carbon of the ring in compounds Va-f.

The oxidation of compounds IVa,c, $f$ and $\mathrm{Va}, \mathrm{c}, \mathrm{f}$ was carried out in refluxing benzene in presence of activated charcoal, afforded 1,6-dihydro-1,2,4,5-tetrazin3-ones Vla,c,f and 6,7-dihydro-1,2,4,5-tetrazepin-6-ones Vlla,c,f respectively, (Figure 3). Both the analytical and spectral data (IR, ${ }^{1} \mathrm{H}$ NMR, ${ }^{13} \mathrm{C}$ NMR and mass spectra) of the title compounds Vla,c,f and Vlla,c,f were in full agreement with the proposed structures and depicted in experimental part. Their mass spectra displayed the correct molecular ion peaks $\left(\mathrm{M}^{+}\right)$in accordance with the suggested structures. Their IR spectra indicating the disappearance of absorption band in the $3435-3220 \mathrm{~cm}^{-1}$ region due to the HN-NH function. In their ${ }^{1} \mathrm{H}$ NMR data, no signals for HN-NH (6.6-6.4 ppm) were observed. Their ${ }^{13} \mathrm{C}$ NMR spectra revealed all the signals of the proposed structures.<smiles>[R]C(=O)C1=NN([Al])C(=O)N([Tl])N1[14CH3]</smiles><smiles>[R]C(=O)C1=NN(Br)C(=O)CN([Tl])N1C</smiles>

Va,c,f<smiles>C[R][C@H](C)c1ccccc1</smiles>

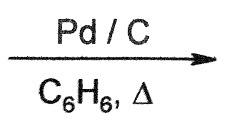<smiles>[R]C(=O)c1nnc(=O)n([Al])n1</smiles>

Vla,c,f<smiles>[R]C(=O)C1=NN([Al])C(=O)CN=N1</smiles>

VIla,c,f

Figure 3. Thermal oxidation of compounds IVa,c,f and Va,c,f 
SOUTH. BRAZ. J. CHEM., Vol.18, No. 18, 2010

H. M. M. Dalloul

\section{EXPERIMENTAL SECTION}

\subsection{General Remarks}

Melting points were determined on thermal melting point apparatus and are uncorrected. The IR spectra were measured as potassium bromide pellets using a Shimadzu FT-IR 8101 PC infrared spectrophotometer. The ${ }^{1} \mathrm{H}$ NMR and ${ }^{13} \mathrm{C}$ NMR spectra were recorded on a Bruker AM $300 \mathrm{MHz}$ spectrometer at room temperature in DMSO- $\mathrm{d}_{6}$ solution using tetramethylsilane (TMS) as internal reference. Chemical shifts were recorded as $\delta$ values in parts per millions (ppm) downfield from internal TMS. Electron impact (EI) mass spectra were run on a GCMS-QP1000 EX spectrometer at $70 \mathrm{eV}$. Elemental analyses were performed at the Microanalytical Center of Cairo University, Egypt. Girardreagent $P$, tetrahydrofuran (THF), benzene and triethylamine were purchased from Avocado Chemical Company, England. Hydrazonoyl halides I [14] were prepared according to the methods reported in the literature.

\subsection{Synthesis of Compounds IVa-f and Va-f}

To a mixture of the appropriate hydrazonoyl halide I $(10 \mathrm{mmol})$ and acetylhydrazide pyridinium chloride III $(10 \mathrm{mmol})$ in dry tetrahydrofuran or 1,4dioxane $(100 \mathrm{~mL})$, triethylamine $(3 \mathrm{ml}, 20 \mathrm{mmol})$ was added at room temperature and the reaction mixture was controlled by TLC. The stirring continued until the starting substrates were completely consumed (12-18 h). The triethylammonium chloride salt was filtered off, the solvent was removed under reduced pressure and the residue was triturated with methanol. The solid thus obtained was filtered off and chromotographed using silica gel TLC, elution with benzene: ethyl acetate: petroleum ether $\left(40-60^{\circ} \mathrm{C}\right)$ 5:4:1 afforded the desired products IVa-f.

The following compounds were prepared using this method:

6-Acetyl-4-(4-chlorophenyl)-1,2,3,4-tetrahydro-1,2,4,5-tetrazin-3-one (IVa): White solid; yield: $67 \%$; Mp. $178-180^{\circ} \mathrm{C}$; ${ }^{1} \mathrm{H}$ NMR $(300 \mathrm{MHz}$, DMSO-d TMS) $\delta: 2.56\left(\mathrm{~s}, 3 \mathrm{H}, \mathrm{CH}_{3}\right), 6.51(\mathrm{~s}, 1 \mathrm{H}, \mathrm{NH}), 6.45(\mathrm{~s}, 1 \mathrm{H}, \mathrm{NH}), 7.10-7.68(\mathrm{~m}, 4 \mathrm{H}$, $\mathrm{Ar}-\mathrm{H}$ ). ${ }^{13} \mathrm{C}$ NMR (300 MHz, DMSO-d 6 , TMS) $\delta: 192.4\left(\mathrm{CH}_{3} \mathrm{C}=\mathrm{O}\right), 171.8$ (ring $\mathrm{C}=\mathrm{O}), 146.8(\mathrm{C}=\mathrm{N}), 141.6-125.1(\mathrm{Ar}-\mathrm{C}), 26.5\left(\mathrm{CH}_{3}\right) . \mathrm{IR}\left(\mathrm{KBr}\right.$ disk, $\left.\mathrm{cm}^{-1}\right): 3430$, $3236(\mathrm{NH}), 1675$ (ring $\mathrm{C}=\mathrm{O}), 1690(\mathrm{C}=\mathrm{O}), 1598(\mathrm{C}=\mathrm{N}) . \quad \mathrm{MS},(\mathrm{m} / \mathrm{z}): 252 / 254[\mathrm{M}]^{+}$ chlorine isotopes. Anal, Calcd for $\mathrm{C}_{10} \mathrm{H}_{9} \mathrm{CIN}_{4} \mathrm{O}_{2}\left(\mathrm{M}_{\mathrm{r}}=252.66\right)$ : $\mathrm{C}, 47.54 ; \mathrm{H}, 3.59$; $\mathrm{N}, 22.17 \%$; Found: $\mathrm{C}, 47.60 ; \mathrm{H}, 3.50 \mathrm{~N}, 22.10 \%$.

6-Benzoyl-4-(4-chlorophenyl)-1,2,3,4-tetrahydro-1,2,4,5-tetrazin-3-one (IVb): White solid, yield: $62 \%$; Mp. $161-163{ }^{\circ} \mathrm{C},{ }^{1} \mathrm{H}$ NMR $(300 \mathrm{MHz}$, DMSO-d TMS) $\delta: 6.52(\mathrm{~s}, 1 \mathrm{H}, \mathrm{NH}), 6.47(\mathrm{~s}, 1 \mathrm{H}, \mathrm{NH}), 7.13-7.72(\mathrm{~m}, 9 \mathrm{H}, \mathrm{Ar}-\mathrm{H}) .{ }^{13} \mathrm{C}$ NMR $\left(300 \mathrm{MHz}, \mathrm{DMSO}-\mathrm{d}_{6}, \mathrm{TMS}\right) \delta: 184.7(\mathrm{PhC}=\mathrm{O}), 171.6$ (ring $\left.\mathrm{C}=0\right), 146.6(\mathrm{C}=\mathrm{N})$, 141.6-121.4 (Ar-C). IR (KBr disk, $\left.\mathrm{cm}^{-1}\right)$ : 3432, $3224(\mathrm{NH}), 1674$ (ring $\mathrm{C}=\mathrm{O}$ ), 1660 $(\mathrm{PhC}=0), 1596(\mathrm{C}=\mathrm{N})$. MS, $\mathrm{m} / \mathrm{z}: 314 / 316[\mathrm{M}]^{+}$chlorine isotopes. Anal, Calcd for $\mathrm{C}_{15} \mathrm{H}_{11} \mathrm{ClN}_{4} \mathrm{O}_{2}\left(\mathrm{M}_{\mathrm{r}}=314.73\right): \mathrm{C}, 57.24 ; \mathrm{H}, 3.52 ; \mathrm{N}, 17.80 \%$; Found: $\mathrm{C}, 57.35 ; \mathrm{H}$, $3.45 \mathrm{~N}, 17.89 \%$.

4-(4-Chlorophenyl)-6-phenylaminocarbonyl-1,2,3,4-tetrahydro1,2,4,5-tetrazin-3-one (IVc): Off white solid, yield: $68 \%$; Mp. $189-191^{\circ} \mathrm{C},{ }^{1} \mathrm{H}$ NMR (300 MHz, DMSO-d $\left.\mathrm{d}_{6}, \mathrm{TMS}\right) \delta: 6.48(\mathrm{~s}, 1 \mathrm{H}, \mathrm{NH}), 6.44(\mathrm{~s}, 1 \mathrm{H}, \mathrm{NH}), 7.13-7.71(\mathrm{~m}$, 


\title{
SOUTH. BRAZ. J. CHEM., Vol.18, No. 18, 2010
}

\author{
Synthesis of Tetrazinone and Tetrazepinone Derivatives
}

$9 \mathrm{H}, \mathrm{Ar}-\mathrm{H}), 10.2(\mathrm{~s}, 1 \mathrm{H}, \mathrm{NH}) .{ }^{13} \mathrm{C}$ NMR (300 MHz, DMSO-d 6 , TMS) $\delta: 171.6$ (ring $\mathrm{C}=\mathrm{O}), 159.7(\mathrm{PhNHC}=\mathrm{O}), 147.6(\mathrm{C}=\mathrm{N}), 141.4-121.6(\mathrm{Ar}-\mathrm{C}) . \mathrm{IR}\left(\mathrm{KBr}\right.$ disk, $\left.\mathrm{cm}^{-1}\right)$ : $3428,3272,3227(\mathrm{NH}), 1672$ (ring $\mathrm{C}=\mathrm{O}), 1655(\mathrm{PhC}=\mathrm{O}), 1599(\mathrm{C}=\mathrm{N}) . \mathrm{MS}, \mathrm{m} / \mathrm{z}$ : $329 / 331[\mathrm{M}]^{+}$chlorine isotopes. Anal, Calcd for $\mathrm{C}_{15} \mathrm{H}_{12} \mathrm{ClN}_{5} \mathrm{O}_{2}\left(\mathrm{M}_{\mathrm{r}}=329.75\right): \mathrm{C}$, $54.64 ; \mathrm{H}, 3.67 ; \mathrm{N}, 21.24 \%$; Found: C, $54.55 ; \mathrm{H}, 3.75 \mathrm{~N}, 21.15 \%$.

4-(4-Chlorophenyl)-6-(2-furoyl)-1,2,3,4-tetrahydro-1,2,4,5-tetrazin-3one (IVd): White solid, yield: $64 \%$; Mp. $147-149^{\circ} \mathrm{C},{ }^{1} \mathrm{H}$ NMR (300 MHz, DMSO$\left.\mathrm{d}_{6}, \mathrm{TMS}\right) \delta: 6.60(\mathrm{~s}, 1 \mathrm{H}, \mathrm{NH}), 6.56(\mathrm{~s}, 1 \mathrm{H}, \mathrm{NH}), 7.16-7.80(\mathrm{~m}, 7 \mathrm{H}, \mathrm{Ar}-\mathrm{H}) .{ }^{13} \mathrm{C}$ NMR (300 MHz, DMSO-d $\left.\mathrm{d}_{6}, \mathrm{TMS}\right) \delta: 174.6(\mathrm{C}=\mathrm{O}), 171.2$ (ring $\left.\mathrm{C}=\mathrm{O}\right), 147.1(\mathrm{C}=\mathrm{N})$, 142.3-121.4 (Ar-C). IR ( $\mathrm{KBr}$ disk, $\left.\mathrm{cm}^{-1}\right)$ : 3433, $3237(\mathrm{NH}), 1674$ (ring $\mathrm{C}=\mathrm{O}$ ), 1665 $(\mathrm{C}=\mathrm{O}), 1612(\mathrm{C}=\mathrm{N})$. MS, $\mathrm{m} / \mathrm{z}: 304 / 306[\mathrm{M}]^{+}$. Anal, Calcd for $\mathrm{C}_{13} \mathrm{H}_{9} \mathrm{ClN}_{4} \mathrm{O}_{3}\left(\mathrm{M}_{\mathrm{r}}=\right.$ 304.69): C, 51.25; H, 2.98; N, 18.39\%; Found: C, $51.35 ; \mathrm{H}, 3.05 \mathrm{~N}, 18.30 \%$.

4-(4-Chlorophenyl)-6-(2-thenoyl)-1,2,3,4-tetrahydro-1,2,4,5-tetrazin-3one (IVe): Yellow solid, yield: $65 \%$; Mp. 195-197 ${ }^{\circ} \mathrm{C},{ }^{1} \mathrm{H}$ NMR (300 MHz, DMSO$\left.\mathrm{d}_{6}, \mathrm{TMS}\right) \delta$ : $6.58(\mathrm{~s}, 1 \mathrm{H}, \mathrm{NH}), 6.53(\mathrm{~s}, 1 \mathrm{H}, \mathrm{NH}), 7.12-7.82(\mathrm{~m}, 7 \mathrm{H}, \mathrm{Ar}-\mathrm{H}) .{ }^{13} \mathrm{C} N M R$ (300 MHz, DMSO-d $\left.\mathrm{d}_{6}, \mathrm{TMS}\right) \delta: 175.4(\mathrm{C}=0), 171.4$ (ring $\left.\mathrm{C}=\mathrm{O}\right), 146.9(\mathrm{C}=\mathrm{N})$, 142.6-121.7 (Ar-C). IR ( $\mathrm{KBr}$ disk, $\mathrm{cm}^{-1}$ ): 3431, $3228(\mathrm{NH}), 1675$ (ring $\mathrm{C}=0$ ), 1660 $(\mathrm{C}=\mathrm{O}), 1610(\mathrm{C}=\mathrm{N})$. MS, m/z: 320/322 [M] chlorine isotopes. Anal, Calcd for $\mathrm{C}_{13} \mathrm{H}_{9} \mathrm{CIN}_{4} \mathrm{O}_{2} \mathrm{~S}\left(\mathrm{M}_{\mathrm{r}}=320.76\right): \mathrm{C}, 48.68 ; \mathrm{H}, 2.83 ; \mathrm{N}, 17.47 \%$; Found: $\mathrm{C}, 48.80 ; \mathrm{H}$, $2.90 \mathrm{~N}, 17.55 \%$.

4-(4-Chlorophenyl)-6-(2-naphthoyl)-1,2,3,4-tetrahydro-1,2,4,5-tetra-

zin-3-one (IVf): Pale yellow solid, yield: $60 \%$; Mp. 201-203 ${ }^{\circ} \mathrm{C},{ }^{1} \mathrm{H}$ NMR (300 $\left.\mathrm{MHz}, \mathrm{DMSO}-\mathrm{d}_{6}, \mathrm{TMS}\right) \delta: 6.60(\mathrm{~s}, 1 \mathrm{H}, \mathrm{NH}), 6.54(\mathrm{~s}, 1 \mathrm{H}, \mathrm{NH}), 7.22-8.42(\mathrm{~m}, 11 \mathrm{H}$, Ar-H). ${ }^{13} \mathrm{C}$ NMR (300 MHz, DMSO-d $\mathrm{d}_{6}$, TMS) $\delta: 184.5(\mathrm{C}=0), 170.8$ (ring $\mathrm{C}=0$ ), $146.7(\mathrm{C}=\mathrm{N}), 142.2-120.9(\mathrm{Ar}-\mathrm{C}) . \mathrm{IR}\left(\mathrm{KBr}\right.$ disk, $\left.\mathrm{cm}^{-1}\right): 3428,3221(\mathrm{NH}), 1675$ (ring $\mathrm{C}=\mathrm{O}), 1650(\mathrm{C}=\mathrm{O}), 1605(\mathrm{C}=\mathrm{N}) . \mathrm{MS},(\mathrm{m} / \mathrm{z}): 364 / 366[\mathrm{M}]^{+}$chlorine isotopes. Anal, Calcd for $\mathrm{C}_{19} \mathrm{H}_{13} \mathrm{CIN}_{4} \mathrm{O}_{2}\left(\mathrm{M}_{\mathrm{r}}=364.79\right): \mathrm{C}, 62.56 \mathrm{H}, 3.59 ; \mathrm{N}, 15.36 \%$; Found: $\mathrm{C}$, $62.45 ; \mathrm{H}, 3.70 \mathrm{~N}, 15.30 \%$.

3-Acetyl-5-(4-chlorophenyl)-1,2,6,7-tetrahydro-1,2,4,5-tetrazepin-6one (Va): White solid, yield: $32 \%$; Mp. $134-136{ }^{\circ} \mathrm{C},{ }^{1} \mathrm{H}$ NMR (300 MHz, DMSO-d TMS) $\delta: 2.54\left(\mathrm{~s}, 3 \mathrm{H}, \mathrm{CH}_{3}\right), 4.34\left(\mathrm{~s}, 2 \mathrm{H}, \mathrm{CH}_{2}\right), 6.50(\mathrm{~s}, 1 \mathrm{H}, \mathrm{NH}), 6.41(\mathrm{~s}, 1 \mathrm{H}, \mathrm{NH})$, 7.10-7.68 (m, 4H). ${ }^{13} \mathrm{C}$ NMR (300 MHz, DMSO-d 6 , TMS) $\delta: 192.4\left(\mathrm{CH}_{3} \mathrm{C}=\mathrm{O}\right)$, 170.8 (ring $\mathrm{C}=\mathrm{O}), 147.8(\mathrm{C}=\mathrm{N}), 142.6-125.6(\mathrm{Ar}-\mathrm{C}), 43.8\left(\mathrm{CH}_{2}\right), 26.6\left(\mathrm{CH}_{3}\right)$. IR $\left(\mathrm{KBr}\right.$ disk, $\left.\mathrm{cm}^{-1}\right)$ : $3430,3236(\mathrm{NH}), 1675$ (ring $\left.\mathrm{C}=\mathrm{O}\right), 1687(\mathrm{C}=\mathrm{O}), 1598(\mathrm{C}=\mathrm{N})$. MS, m/z: $266 / 268[\mathrm{M}]^{+}$chlorine isotopes. Anal, Calcd for $\mathrm{C}_{11} \mathrm{H}_{11} \mathrm{CIN}_{4} \mathrm{O}_{2}\left(\mathrm{M}_{\mathrm{r}}=\right.$ 266.69): C, 49.54; H, 4.16; N, 21.01\%; Found: C, 49.65; H, 4.05 N, 20.90\%.

3-Benzoyl-5-(4-chlorophenyl)-1,2,6,7-tetrahydro-1,2,4,5-tetrazepin-6one (Vb): White solid, yield: $31 \%$; Mp. $154-156^{\circ} \mathrm{C},{ }^{1} \mathrm{H}$ NMR $\left(300 \mathrm{MHz}, \mathrm{DMSO}-\mathrm{d}_{6}\right.$, TMS) $\delta: 4.33\left(\mathrm{~s}, 2 \mathrm{H}, \mathrm{CH}_{2}\right), 6.47(\mathrm{~s}, 1 \mathrm{H}, \mathrm{NH}), 6.43(\mathrm{~s}, 1 \mathrm{H}, \mathrm{NH}), 7.13-7.72(\mathrm{~m}, 9 \mathrm{H}$, $\mathrm{Ar}-\mathrm{H}) .{ }^{13} \mathrm{C}$ NMR (300 MHz, DMSO-d 6 , TMS) $\delta: 184.7(\mathrm{PhC}=0), 171.6$ (ring $\mathrm{C}=0$ ), $146.6(\mathrm{C}=\mathrm{N}), 141.6-121.4(\mathrm{Ar}-\mathrm{C}), 43.7\left(\mathrm{CH}_{2}\right)$. IR $\left(\mathrm{KBr}\right.$ disk, $\left.\mathrm{cm}^{-1}\right): 3430,3236$ $(\mathrm{NH}), 1674$ (ring $\mathrm{C}=\mathrm{O}$ ), $1665(\mathrm{PhC}=\mathrm{O}), 1596(\mathrm{C}=\mathrm{N}) . \quad \mathrm{MS}, \mathrm{m} / \mathrm{z}: 328 / 330[\mathrm{M}]^{+}$ chlorine isotopes. Anal, Calcd for $\mathrm{C}_{16} \mathrm{H}_{13} \mathrm{CIN}_{4} \mathrm{O}_{2}\left(\mathrm{M}_{\mathrm{r}}=328.76\right): \mathrm{C}, 58.46 ; \mathrm{H}, 3.99$; N, 17.04\%; Found: C, 58.36; $\mathrm{H}, 4.07 \mathrm{~N}, 16.95 \%$.

5-(4-Chlorophenyl)-3-phenylaminocarbonyl-1,2,6,7-tetrahydro-1,2,4, 5-tetrazepin-6-one (Vc): White solid, yield: $30 \%$; Mp. 179-181 ${ }^{\circ} \mathrm{C},{ }^{1} \mathrm{H}$ NMR (300 $\mathrm{MHz}$, DMSO-d $\mathrm{d}_{6}$, TMS) $\delta: 4.32\left(\mathrm{~s}, 2 \mathrm{H}, \mathrm{CH}_{2}\right), 6.48(\mathrm{~s}, 1 \mathrm{H}, \mathrm{NH}), 6.42(\mathrm{~s}, 1 \mathrm{H}, \mathrm{NH})$, 7.13-7.71 (m, 9H, Ar-H), $10.3(\mathrm{~s}, 1 \mathrm{H}, \mathrm{NH}) .{ }^{13} \mathrm{C}$ NMR (300 MHz, DMSO-d 6 , TMS) $\delta$ : 
$184.7(\mathrm{PhC}=\mathrm{O}), 171.6$ (ring $\mathrm{C}=\mathrm{O}), 159.7(\mathrm{PhNHC}=\mathrm{O}), 147.6(\mathrm{C}=\mathrm{N}), 141.4-121.6$ (Ar-C), $43.5\left(\mathrm{CH}_{2}\right)$. IR ( $\mathrm{KBr}$ disk, $\left.\mathrm{cm}^{-1}\right)$ : 3430, 3275, $3236(\mathrm{NH}), 1672$ (ring $\mathrm{C}=\mathrm{O}$ ), $1655(\mathrm{PhC}=\mathrm{O}), 1599(\mathrm{C}=\mathrm{N}) . \mathrm{MS}, \mathrm{m} / \mathrm{z}: 343 / 345[\mathrm{M}]^{+}$chlorine isotopes. Anal, Calcd for $\mathrm{C}_{16} \mathrm{H}_{14} \mathrm{CIN}_{5} \mathrm{O}_{2}\left(\mathrm{M}_{\mathrm{r}}=343.78\right)$ : $\mathrm{C}, 55.90 ; \mathrm{H}, 4.10 ; \mathrm{N}, 20.37 \%$; Found: $\mathrm{C}, 55.88$; $\mathrm{H}, 3.91 \mathrm{~N}, 20.25 \%$.

5-(4-Chlorophenyl)-3-(2-furoyl)-1,2,6,7-tetrahydro-1,2,4,5-tetrazepin6-one (Vd): White solid, yield: $33 \%$; Mp. $149-151^{\circ} \mathrm{C},{ }^{1} \mathrm{H}$ NMR (300 MHz, DMSO$\left.\mathrm{d}_{6}, \mathrm{TMS}\right) \delta: 4.30\left(\mathrm{~s}, 2 \mathrm{H}, \mathrm{CH}_{2}\right), 6.52(\mathrm{~s}, 1 \mathrm{H}, \mathrm{NH}), 6.47(\mathrm{~s}, 1 \mathrm{H}, \mathrm{NH}), 7.16-7.80(\mathrm{~m}$, $7 \mathrm{H}, \mathrm{Ar}-\mathrm{H}) .{ }^{13} \mathrm{C}$ NMR (300 MHz, DMSO-d 6 , TMS) $\delta: 174.6(\mathrm{C}=\mathrm{O}), 171.2$ (ring $\mathrm{C}=\mathrm{O}), 147.1(\mathrm{C}=\mathrm{N}), 142.3-121.4(\mathrm{Ar}-\mathrm{C}), 43.6\left(\mathrm{CH}_{2}\right)$. IR $\left(\mathrm{KBr}\right.$ disk, $\left.\mathrm{cm}^{-1}\right): 1674$ (ring $\mathrm{C}=\mathrm{O}), 1665(\mathrm{C}=\mathrm{O}), 1612(\mathrm{C}=\mathrm{N})$. MS, $\mathrm{m} / \mathrm{z}: 318 / 320[\mathrm{M}]^{+}$chlorine isotopes. Anal, Calcd for $\mathrm{C}_{14} \mathrm{H}_{11} \mathrm{CIN}_{4} \mathrm{O}_{3}\left(\mathrm{M}_{\mathrm{r}}=318.72\right): \mathrm{C}, 52.76 ; \mathrm{H}, 3.48 ; \mathrm{N}, 17.58 \%$; Found: $\mathrm{C}$, $52.65 ; \mathrm{H}, 3.40 \mathrm{~N}, 17.65 \%$.

5-(4-Chlorophenyl)-3-(2-thenoyl)-1,2,6,7-tetrahydro-1,2,4,5-tetrazepin-6-one (Ve): White solid, yield: $31 \%$; Mp. $171-173{ }^{\circ} \mathrm{C},{ }^{1} \mathrm{H}$ NMR $(300 \mathrm{MHz}$, DMSO-d 6 , TMS) $\delta: 4.33\left(\mathrm{~s}, 2 \mathrm{H}, \mathrm{CH}_{2}\right), 6.50(\mathrm{~s}, 1 \mathrm{H}, \mathrm{NH}), 6.46(\mathrm{~s}, 1 \mathrm{H}, \mathrm{NH}), 7.12-$ $7.82(\mathrm{~m}, 7 \mathrm{H}, \mathrm{Ar}-\mathrm{H}) .{ }^{13} \mathrm{C}$ NMR $\left(300 \mathrm{MHz}\right.$, DMSO-d $\left.\mathrm{d}_{6}, \mathrm{TMS}\right) \delta: 175.4(\mathrm{C}=\mathrm{O}), 171.4$ (ring $\mathrm{C}=\mathrm{O}), 146.9(\mathrm{C}=\mathrm{N}), 142.6-121.7(\mathrm{Ar}-\mathrm{C}), 43.5\left(\mathrm{CH}_{2}\right)$. IR $\left(\mathrm{KBr}\right.$ disk, $\left.\mathrm{cm}^{-1}\right)$ : 3430, $3236(\mathrm{NH}), 1675$ (ring $\mathrm{C}=\mathrm{O}), 1660(\mathrm{C}=\mathrm{O}), 1610(\mathrm{C}=\mathrm{N})$. MS, $\mathrm{m} / \mathrm{z}: 334 / 336$ $[\mathrm{M}]^{+}$chlorine isotopes. Anal, Calcd for $\mathrm{C}_{14} \mathrm{H}_{11} \mathrm{ClN}_{4} \mathrm{O}_{2} \mathrm{~S}\left(\mathrm{M}_{\mathrm{r}}=334.79\right)$ : $\mathrm{C}, 50.23$; $\mathrm{H}, 3.31 ; \mathrm{N}, 16.74 \%$; Found: $\mathrm{C}, 50.30 ; \mathrm{H}, 3.21 \mathrm{~N}, 16.65 \%$.

5-(4-chlorophenyl)-3-(2-naphthoyl)-1,2,6,7-tetrahydro-1,2,4,5-tetrazepin-6-one (Vf): White solid, yield: $28 \%$; Mp. $162-164{ }^{\circ} \mathrm{C},{ }^{1} \mathrm{H}$ NMR $(300 \mathrm{MHz}$, DMSO-d $\left.\mathrm{d}_{6}, \mathrm{TMS}\right) \delta: 4.32\left(\mathrm{~s}, 2 \mathrm{H}, \mathrm{CH}_{2}\right), 6.45(\mathrm{~s}, 1 \mathrm{H}, \mathrm{NH}), 6.41(\mathrm{~s}, 1 \mathrm{H}, \mathrm{NH}), 7.22-$ $8.42(\mathrm{~m}, 11 \mathrm{H}, \mathrm{Ar}-\mathrm{H}) .{ }^{13} \mathrm{C}$ NMR $\left(300 \mathrm{MHz}, \mathrm{DMSO}-\mathrm{d}_{6}, \mathrm{TMS}\right) \delta: 184.5(\mathrm{C}=\mathrm{O}), 170.8$ (ring $\mathrm{C}=\mathrm{O}), 146.7(\mathrm{C}=\mathrm{N}), 142.2-120.9(\mathrm{Ar}-\mathrm{C}), 43.7\left(\mathrm{CH}_{2}\right)$. IR $\left(\mathrm{KBr}\right.$ disk, $\left.\mathrm{cm}^{-1}\right)$ : $3430,3236(\mathrm{NH}), 1675$ (ring $\mathrm{C}=\mathrm{O}), 1650(\mathrm{C}=\mathrm{O}), 1605(\mathrm{C}=\mathrm{N}) . \mathrm{MS}, \mathrm{m} / \mathrm{z}: 378 / 380$ $[\mathrm{M}]^{+}$chlorine isotopes. Anal, Calcd for $\mathrm{C}_{20} \mathrm{H}_{15} \mathrm{ClN}_{4} \mathrm{O}_{2}\left(\mathrm{M}_{\mathrm{r}}=378.82\right): \mathrm{C}, 63.41 ; \mathrm{H}$, 3.99 ; N, 14.79\%; Found: C, $63.30 ; \mathrm{H}, 4.10 \mathrm{~N}, 14.85 \%$.

\subsection{Thermal Oxidation of Compounds $4 a, c, f$ and $5 a, c, f$}

A compounds $4 a, c, f$ or $5 a, c, f(5 \mathrm{mmol})$ and $\mathrm{Pd} / \mathrm{charcoal}$ in benzene $(50 \mathrm{~mL})$ were heated to reflux for 2-4 hours and monitored by TLC. The reaction mixture was cooled, then filtered and the solvent evaporated. The residual solid was collected and recrystallized from ethanol to give the desired compounds $4 a, c, f$ and $5 a, c, f$. The following compounds were prepared using this method:

3-Acetyl-5-(4-chlorophenyl)-1,6-dihydro-1,2,4,5-tetrazin-3-ones (Vla): White solid, yield: $77 \%$; Mp. $230-232{ }^{\circ} \mathrm{C},{ }^{1} \mathrm{H}$ NMR $(300 \mathrm{MHz}$, DMSO-d 6 , TMS) $\delta$ : 2.54 (s, 3H, $\left.\mathrm{CH}_{3}\right), 7.10-7.68(\mathrm{~m}, 4 \mathrm{H}, \mathrm{Ar}-\mathrm{H}) .{ }^{13} \mathrm{C}$ NMR (300 MHz, DMSO-d 6 , TMS) $\delta$ : $192.8(\mathrm{RC}=\mathrm{O}), 172.5$ (ring $\mathrm{C}=\mathrm{O}), 147.8(\mathrm{C}=\mathrm{N}), 142.6-126.0(\mathrm{Ar}-\mathrm{C}), 26.7\left(\mathrm{CH}_{3}\right)$. IR $\left(\mathrm{KBr}\right.$ disk, $\left.\mathrm{cm}^{-1}\right): 1690(\mathrm{RC}=\mathrm{O}), 1675$ (ring $\left.\mathrm{C}=\mathrm{O}\right), 1610(\mathrm{C}=\mathrm{N}) . \quad \mathrm{MS}, \mathrm{m} / \mathrm{z}$ : 250/252 [M] $]^{+}$chlorine isotopes. Anal, Calcd for $\mathrm{C}_{10} \mathrm{H}_{7} \mathrm{ClN}_{4} \mathrm{O}_{2}\left(\mathrm{M}_{\mathrm{r}}=250.65\right): \mathrm{C}$, 47.92; H, 2.82; N, 22.35\%; Found: C, $47.80 ; \mathrm{H}, 2.90$ N, $22.25 \%$.

5-(4-Chlorophenyl)-3-phenylaminocarbonyl-1,6-dihydro-1,2,4,5-

tetrazin-3-ones (Vlc): White solid, yield: $54 \%$; Mp. $230-232{ }^{\circ} \mathrm{C},{ }^{1} \mathrm{H}$ NMR $(300$ MHz, DMSO-d 6 , TMS) $\delta: 7.10-7.68(\mathrm{~m}, 9 \mathrm{H}, \mathrm{Ar}-\mathrm{H}), 9.96(\mathrm{~s}, 1 \mathrm{H}, \mathrm{NH}) .{ }^{13} \mathrm{C}$ NMR $(300$ MHz, DMSO-d $\left.\mathrm{d}_{6}, \mathrm{TMS}\right) \delta$ : 172.3 (ring $\left.\mathrm{C}=\mathrm{O}\right), 160.3(\mathrm{PhNHC}=\mathrm{O}), 147.3(\mathrm{C}=\mathrm{N})$, 
SOUTH. BRAZ. J. CHEM., Vol.18, No. 18, 2010

142.3-126.2 (Ar-C). IR ( $\mathrm{KBr}$ disk, $\left.\mathrm{cm}^{-1}\right)$ : 3277 (NH), 1672 (ring $\mathrm{C}=\mathrm{O}$ ), 1660 $(\mathrm{RC}=\mathrm{O}), 1615(\mathrm{C}=\mathrm{N})$. MS, $\mathrm{m} / \mathrm{z}$ : $327 / 329[\mathrm{M}]^{+}$chlorine isotopes. Anal, Calcd for $\mathrm{C}_{15} \mathrm{H}_{10} \mathrm{ClN}_{5} \mathrm{O}_{2}\left(\mathrm{M}_{\mathrm{r}}=327.73\right): \mathrm{C}, 54.97 ; \mathrm{H}, 3.08 ; \mathrm{N}, 21.37 \%$; Found: $\mathrm{C}, 55.10 ; \mathrm{H}$, $2.95 \mathrm{~N}, 21.25 \%$.

5-(4-chlorophenyl)-3-(2-naphthoyl)-1,6-dihydro-1,2,4,5-tetrazin-3ones (VIf): White solid, yield: $76 \%$; Mp. $230-232{ }^{\circ} \mathrm{C},{ }^{1} \mathrm{H}$ NMR (300 MHz, DMSO$\left.\mathrm{d}_{6}, \mathrm{TMS}\right) \delta: 7.10-7.68(\mathrm{~m}, 11 \mathrm{H}, \mathrm{Ar}-\mathrm{H}) .{ }^{13} \mathrm{C}$ NMR $\left(300 \mathrm{MHz}, \mathrm{DMSO}-\mathrm{d}_{6}, \mathrm{TMS}\right) \delta$ : $185.2(\mathrm{RC}=\mathrm{O}), 171.7$ (ring $\mathrm{C}=\mathrm{O}), 147.7(\mathrm{C}=\mathrm{N}), 143.4-121.8(\mathrm{Ar}-\mathrm{C})$. IR $(\mathrm{KBr}$ disk, $\mathrm{cm}^{-1}$ ): 1675 (ring $\left.\mathrm{C}=\mathrm{O}\right), 1650(\mathrm{RC}=\mathrm{O}), 1610(\mathrm{C}=\mathrm{N}) . \mathrm{MS}, \mathrm{m} / \mathrm{z}: 362 / 364[\mathrm{M}]^{+}$. Anal, Calcd for $\mathrm{C}_{19} \mathrm{H}_{11} \mathrm{CIN}_{4} \mathrm{O}_{2}\left(\mathrm{M}_{\mathrm{r}}=362.78\right): \mathrm{C}, 62.91 ; \mathrm{H}, 3.06 ; \mathrm{N}, 15.44 \%$; Found: $\mathrm{C}$, $62.80 ; \mathrm{H}, 2.95 \mathrm{~N}, 15.55 \%$.

3-Acetyl-5-(4-chlorophenyl)-6,7-tetrahydro-1,2,4,5-tetra-zepin-6-one (VIla): White solid, yield: 74\%; Mp. 201-203 ${ }^{\circ} \mathrm{C},{ }^{1} \mathrm{H}$ NMR (300 MHz, DMSO-d TMS) $\delta: 4.44\left(\mathrm{~s}, 2 \mathrm{H}, \mathrm{CH}_{2}\right), 7.22-8.42(\mathrm{~m}, 4 \mathrm{H}, \mathrm{Ar}-\mathrm{H}) .{ }^{13} \mathrm{C}$ NMR $(300 \mathrm{MHz}$, DMSO$\left.\mathrm{d}_{6}, \mathrm{TMS}\right) \delta: 192.0(\mathrm{C}=\mathrm{O}), 172.2$ (ring $\left.\mathrm{C}=\mathrm{O}\right), 148.3(\mathrm{C}=\mathrm{N}), 142.9-124.8($ Ar-C), 44.7 $\left(\mathrm{CH}_{2}\right), 26.7\left(\mathrm{CH}_{3}\right)$. IR $\left(\mathrm{KBr}\right.$ disk, $\left.\mathrm{cm}^{-1}\right): 1693(\mathrm{C}=\mathrm{O}), 1675$ (ring $\left.\mathrm{C}=\mathrm{O}\right), 1605(\mathrm{C}=\mathrm{N})$. MS, $\mathrm{m} / \mathrm{z}$ : $264 / 266[\mathrm{M}]^{+}$chlorine isotopes. Anal, Calcd for $\mathrm{C}_{11} \mathrm{H}_{9} \mathrm{ClN}_{4} \mathrm{O}_{2}\left(\mathrm{M}_{\mathrm{r}}=\right.$ 264.67): C, 49.92; H, 3.43; N, 21.17\%; Found: C, 50.05; H, 3.50 N, 21.10\%.

5-(4-Chlorophenyl)-3-phenylaminocarbonyl-6,7-tetrahydro-1,2,4,5tetra-zepin-6-one (VIIc): White solid, yield: 71\%; Mp. 201-203 ${ }^{\circ} \mathrm{C},{ }^{1} \mathrm{H}$ NMR (300 $\mathrm{MHz}$, DMSO-d 6 , TMS) $\delta: 4.43\left(\mathrm{~s}, 2 \mathrm{H}, \mathrm{CH}_{2}\right), 7.22-8.42(\mathrm{~m}, 9 \mathrm{H}, \mathrm{Ar}-\mathrm{H}), 9.98(\mathrm{~s}, 1 \mathrm{H}$, $\mathrm{NH}$ ). ${ }^{13} \mathrm{C}$ NMR (300 MHz, DMSO-d 6 , TMS) $\delta$ : 172.8 (ring $\mathrm{C}=0$ ), 160.5 $(\mathrm{PhNHC}=\mathrm{O}), 147.7(\mathrm{C}=\mathrm{N}), 143.2-124.9(\mathrm{Ar}-\mathrm{C}), 44.2\left(\mathrm{CH}_{2}\right)$. IR $\left(\mathrm{KBr}\right.$ disk, $\left.\mathrm{cm}^{-1}\right)$ : $3273(\mathrm{NH}), 1673$ (ring $\mathrm{C}=\mathrm{O}), 1655(\mathrm{C}=\mathrm{O}), 1610(\mathrm{C}=\mathrm{N})$. MS, m/z: 341/343 [M] chlorine isotopes. Anal, Calcd for $\mathrm{C}_{16} \mathrm{H}_{12} \mathrm{ClN}_{5} \mathrm{O}_{2}\left(\mathrm{M}_{\mathrm{r}}=341.76\right): \mathrm{C}, 53.23 ; \mathrm{H}, 3.54$; N, 20.49\%; Found: C, 53.35; H, 3.60 N, 20.55\%.

5-(4-chlorophenyl)-3-(2-naphthoyl)-6,7-tetrahydro-1,2,4,5-tetra-zepin6-one (Vlif): White solid, yield: $70 \%$; Mp. 201-203 ${ }^{\circ} \mathrm{C},{ }^{1} \mathrm{H}$ NMR (300 MHz, DMSO$\left.\mathrm{d}_{6}, \mathrm{TMS}\right) \delta: 4.46\left(\mathrm{~s}, 2 \mathrm{H}, \mathrm{CH}_{2}\right), 7.22-8.42(\mathrm{~m}, 11 \mathrm{H}, \mathrm{Ar}-\mathrm{H}) .{ }^{13} \mathrm{C}$ NMR $(300 \mathrm{MHz}$, DMSO- $_{6}$, TMS$) \delta: 184.8(\mathrm{C}=\mathrm{O}), 172.4($ ring $\mathrm{C}=\mathrm{O}), 147.4(\mathrm{C}=\mathrm{N}), 143.6-121.4(\mathrm{Ar}-$ C) $44.8\left(\mathrm{CH}_{2}\right)$. IR $\left(\mathrm{KBr}\right.$ disk, $\left.\mathrm{cm}^{-1}\right)$ : 1675 (ring $\left.\mathrm{C}=\mathrm{O}\right), 1650(\mathrm{C}=\mathrm{O}), 1600(\mathrm{C}=\mathrm{N})$. MS, m/z: $376 / 378[\mathrm{M}]^{+}$chlorine isotopes. Anal, Calcd for $\mathrm{C}_{20} \mathrm{H}_{13} \mathrm{CIN}_{4} \mathrm{O}_{2}\left(\mathrm{M}_{\mathrm{r}}=\right.$ 376.81): C, $63.75 ; \mathrm{H}, 5.48$; N, 14.87\%; Found: C, $63.80 ; \mathrm{H}, 5.55 \mathrm{~N}, 14.95 \%$.

\section{CONCLUSION}

In conclusion, the cyclocondensation of several nitrilimines with Girardreagent $P$ leads to formation of 1,2,3,4-tetrahydro-1,2,4,5-tetrazin-3-ones IVa-f and 1,2,6,7-tetrahydro-1,2,4,5-tetrazepin-6-ones Va-f, which thermally oxidized in presence of activated charcoal to 1,6-dihydro-1,2,4,5-tetrazin-3-ones Vla,c,f and 6,7-dihydro-1,2,4,5-tetrazepin-6-ones Vlla,c,f. 


\section{REFERENCES}

[1] W. R. Guo, X. H. Wei, Bioorg. Med. Chem. Lett. 16, 3702-3704 (2006).

[2] P. Sharma, A. Kumar, v. Sahu, J. Singh, Arkivoc, xii, 218-225 (2008).

[3] G. W. Rao, W. X. Hu, Bioorg. Med. Chem. Lett. 15, 3174-3176 (2005).

[4] W. X. Hu, G. W. Rao, Y. Q. Sun, Bioorg. Med. Chem. Lett. 14, 1177-1181 (2004).

[5] Langnel, D. A. F.; Arrowsmith, J.; Stevens, M. F. G. Arkivoc iii, 421-437 (2000).

[6] A. R. Katrizky, Advances in Heterocyclic Chemistry, Taylors Ed., (1984), pp. $1185-8$.

[7] H. M. Dalloul, P. H. Boyle, Heterocycl. Commun. 9(5), 507-514 (2003).

[8] H. M. Dalloul, H. M. Abu-Shawish, Org. Commun. 1, 1-8 (2008).

[9] H. M. Dalloul, E. A. Mohamed, A. Z. El-Shorafa, Z. Naturforsch. 63b, 585$591(2008)$.

[10] M. R. Ottensooser, Bull. Soc. Chim. 4(45), 1013 (1929); Chem. Abstr. 24, 3755 (1930).

[11] G. S. Siduh, G. Thyagarajan, N. Rao, Tetrahedron Lett., 24, 1637-1638 (1963).

[12] H. M. Dalloul, Tetrahedron, 65, 8722-8726 (2009).

[13] N. H. Al-Said, Z. N. Ishtaiwi, Acta Chim. Slov. 52, 328-331 (2005).

[14] M. M. El-Abadelah, A. Q. Hussein, B. A. Thaher, Heterocycles 32, 1879-1895 (1991).

[15] M. M. El-Abadelah, A. Q. Hussein, M. R. Kamal, K. H. Al-Adhami, Heterocycles 27, 917-924 (1988).

[16] H. M. Hassaneen, A. S. Shawali, N. M. Elwan, N. M. Abounada, Org. Prep. Proced. Int., 24, 171-175 (1992).

[17] P. Frohberg, G. Drutkowski, C. Wagner, Eur. J. Org. Chem., 1654-1663 (2002).

[18] A. M. Farag, M. S. Algharib, Org. Prep. Proced. Int. 20, 521-526 (1988).

VISIT OUR SITE: http://www.sbjchem.he.com.br 